\title{
A GRAPHITE CATHODE DISH
}

\section{BY J. W. TURRENTINE}

In conducting a laboratory course in applied electrochemistry, one devoted largely to that phase of electrochemistry which has to do with solutions and the electrolysis of solutions, the need has been experienced for some good material which could be substituted for platinum in the construction of insoluble electrodes. Platinum, to be sure, makes the ideal insoluble electrode, but its cost makes the matter of supplying a large class with electrodes constructed of it impracticable. The initial expense is great and the care of it is a burden on the instructor.

In the Cornell laboratories, for ordinary work graphite has been substituted. Trouble was experienced with this material on account of the fact that it was porous and absorbed the solutions in which it was used and therefore could not be cleaned readily. This prevented its use in experiments where the impurities might interfere with the desired reaction. Also, at high current densities, the graphite electrodes showed a tendency to disintegrate and to fill the solution with clouds of finely divided graphite powder. This was due, possibly, to the discharge within its pores and beneath its surface of gases which forced off the surface particles. A further objection was found in the graphite's propensity for rubbing off and smutting.

After experimentation to determine a means of preventing the absorption of solutions by the graphite, it was found that if the graphite electrodes were treated while hot with molten paraffine, in amounts which are readily absorbed by then, the surface of the graphite acts as though it were greased and is not wetted by aqueous solutions. Because of surface tension phenomena, the pores are not entered by the solutions. This treatment, by keeping solutions from the pores, should also prevent the disintegration of the electrodes at high current densities; however, whether it does or 
not has yet to be determined. Certainly it does not when the graphite is made anode in chloride solutions, but here we have the paraffine attacked by the evolved chlorine and naturally its efficiency as a protective agent is diminished. The treatment with paraffine furthermore reduces to a minimum the inclination of the graphite to rub off, so that it is rendered quite clean and it may then be handled freely without smutting.

When mention was made to the Acheson Graphite Company of the behavior of the graphite when thus treated, it was found that they have employed the same means for improving their electrodes when designed for certain purposes.

In the Wesleyan laboratory we are now using graphite electrodes made from the Acheson sheet graphite of $0.5 \mathrm{~cm}$ thickness. The sheet is cut into pieces which are $15 \mathrm{~cm}$ by $2.5 \mathrm{~cm}$ in dimensions. One end is copper plated and a copper wire about $20 \mathrm{~cm}$ long is twisted around this end and is soldered in place to the copper plate. To protect the exposed metal at this point from fumes, the end of the electrode is covered with a light coating of varnish. The graphite is then paraffined, while hot. The electrodes are supported by a very simple device which consists merely of a block of wood of sufficient size to rest across the top of a beaker. It contains two parallel slits, $2.75 \mathrm{~cm}$ apart and of the proper width to admit the electrodes and to hold them snugly in position.

We were thus enabled to supplant very satisfactorily by the use of paraffined graphite the platinum sheet electrodes heretofore used in the ordinary experiments in the laboratory work. In electrochemical analysis, however, and separations, expediency still demanded the use of the Classen platinum dish.

The use of a graphite dish as a substitute for the platinum dish need not be confined to pedagogic electrochemistry, but should also find an extended use in commerical laboratories.

Recent developments in the field of electrochemical 
analysis, due to the important work of Professor E. F. Smith and students and other investigators, have afforded us many beautiful methods of electrochemical analysis which might well and should be used in industrial laboratories. This is especially true of those laboratories connected with metallurgical plants where some electrochemical methods are already in use. The substitution of the very rapid new methods for those already in vogue, requiring hours for precipitations and separations which could be accomplished in a few minutes if accompanied by effective stirring, would perhaps recommend itself to the works chemist if this substitution could be made without the very considerable outlay now necessary for platinum electrodes. The very convenient Classen dish is not essential to the equipment requisite for electro-analysis, yet it is almost a necessity; one's equipment is scarcely complete without a number of them. To provide them, however, requires a considerable outlay.

By changing from the old system of electrochemical analysis to the new a saving in the amount of platinum in use could undoubtedly be effected. The old system involves the use of the stationary, cylindrical, platinum cathodes, while the new methods depend on rotating electrodes, stirred solutions, the platinum dish and other devices for making possible the use of high current densities. The only points that can be produced in favor of the stationary electrode method is that the electrolysis can be started in the late afternoon and without further attention may be allowed to complete itself during the night, and that the multiplication of the number of analyses does not involve an equal increment in the time required for the operation. However, increasing the number of analyses does increase the number of electrodes which are necessary and therefore increases the cost for equipment. With a single rotating anode and Classen dish cathode the number of analyses could be made with a time expenditure of only a few minutes for each analysis and would require the attention of only a fairly expert chemist.

In equipping a new laboratory for electrochemical anal- 
ysis it is clearly poor economy to install the antiquated apparatus, numerous stationary electrodes, in preference to the new, a much smaller number of rotating anodes and dish cathodes; and there are those who think, with reasons, that it is also poor chemistry.

If our contention is true that it is both good economy and good analytical chemistry not only to equip laboratories with the apparatus requisite for performing rapid analyses in stirred solutions, but also, if necessary, to discard present equipment of stationary electrodes in order to do so, then much more true is our contention if an apparatus constructed of a much less expensive material than platinum can be offered the profession.

Anticipating these advantages and encouraged by the success of the paraffined graphite electrodes, a graphite dish was prepared from a four inch, cylindrical stick of Acheson graphite. This dish was turned out on a lathe by Mr. G. B. Upton, of Sibly College, Cornell University, for whose great kindness I take this opportunity of expressing my sincere appreciation.

Graphite was chosen as the material of which to construct the dish because in it we have a substance which is at once a good conductor of the electric current, is cheap, is light in weight and is non-attackable by solutions. Its density is low, a little less than $2.2,^{1}$ but varies from one electrode to another, and in different parts of a single electrode. With the density probably also varies the hardness, though the variation in both density and hardness is slight and gradual, so no difficulty on that account need be experienced in machining. Because of its low density, the walls of a dish may be made of sufficient thickness to lend strength and rigidity to the article without unduly increasing its weight.

The porosity of the graphite may prove to be the greatest obstacle in the way of the dish's becoming a success when

${ }^{1}$ FitzGerald: Trans. Am. Electrochem. Soc., Vol. II, I909. 
employed in the most accurate analysis. The degree of porosity is expressed numerically as the quotient obtained by dividing the difference between the real and apparent specific gravities by the real specific gravity. ${ }^{1}$ The apparent specific gravity is that obtained when the interstices of the graphite are kept free of the liquid in which the determination is being made. This is accomplished by coating the sample lightly with shellac. The real specific gravity is found after the air has been removed from the pores of the sample by greatly reducing the pressure over the immersing liquid. When the suction is released the evacuated pores become filled. The average of several determinations gives the porosity of graphite at about 0.25. Such a degree of porosity would seem to make this substance an impossible one of which to construct a dish designed for such purposes as is this one. Very effective, however, is the paraffine in preventing the absorption of solutions into its pores. That the latter are not entirely filled by the paraffine is shown by the fact that alcohol is able to penetrate the paraffined graphite fairly readily. It is highly probable that while aqueous solutions do not enter the interstices, aqueous vapor does and condenses there to a certain extent; so it may be necessary eventually to fill these entirely with paraffine by the evacuation method used in the specific gravity determination.

The purity of graphite is high and therefore it is improbable that any contamination of solutions can possibly result from its use. It has been shown ${ }^{2}$ that when a carbonaceous substance, like anthracite coal, has been graphitized, its impurities are almost completely volatilized, the more complete being the volatilization the higher the temperature of graphitization and the longer this heat is maintained. Graphite is then a very pure form of carbon and the Acheson product, in addition to its insolubility, has been shown ${ }^{3}$ to be

\footnotetext{
${ }^{1}$ Fitzgerald: Trans. Am. Electrochem. Soc., Vol. II, Igog.

2 Fitzgerald: Jour. Franklin Inst., Nov., I 902.

3 Foerster: Zeit, angew. Chem., June, IgoI.
} 
very resistant to disintegration in solution. When made anode in caustic solutions, it is only very slightly attacked by the evolved oxygen. This is to be expected when we recall that the combustion point of some forms of graphite in oxygen is about $600^{\circ} \mathrm{C}$.

Graphite is to be had of varying degrees of hardness, the harder varieties, while less pure, are more resistant to chemical action and undoubtedly would make a stronger and more durable article. Hardness would likewise enhance the possibilities for accurate and sharp machining, a fact to be borne in mind when choosing a graphite for the purposes described in this paper. Its tensile strength is about 80 percent of that of the carbon from which it is made, there being a deterioration of 20 percent in that property during the process of manufacture. With the combination of hardness and the above tensile strength it is possible to machine a dish whose walls are of very slight and uniform thickness, which thus possesses both strength and lightness in weight. The elasticity of the harder varieties of graphite is also worthy of note. Possessing these properties, there is no reason then why a graphite dish with proper use should not prove to be long lived to an entirely satisfactory extent.

The experimental dish was prepared for use by first burnishing on a revolving, steel-wire buffer, which served to remove all dust and gave the surface a somewhat polished appearance. The graphite still rubbed off on the fingers, but to a much lessened extent; after treating with paraffine it no longer rubbed off at all.

The dish was warmed in a hot air bath to a temperature of about $110^{\circ}$; while the dish was still hot molten paraffine was applied with a brush until it was no longer absorbed readily but remained visible on the surface. The dish was then returned to the oven, several sheets of filter paper were placed under it and the bath was maintained at the temperature of $I 10^{\circ}$ for several hours. In this way the excess of paraffine was drawn off, by gravity and capillary action, into the filter paper. After this treatment the surface of the 
graphite appeared quite dry. The dish was then reburnished.

The preliminary experiments conducted with this dish gave such very promising results that two other dishes were obtained through the esteemed courtesy of Messrs. Eimer \& Amend, of New York. These are much lighter in weight, weighing respectively 38 and 32 grams after having been paraffined. Their capacity is $200 \mathrm{cc}$. In dimensions they are $9.5 \mathrm{~cm}$ in diameter across the top and are $5 \mathrm{~cm}$ deep. The lower part has the shape of a truncated cone, the conical shape beginning $2.5 \mathrm{~cm}$ from the upper edge and extending inward at such an angle that a flat bottom of $3.5 \mathrm{~cm}$ in diameter is afforded by the truncation. This shape was chosen so as to give a dish of straight sides. The walls are slightly less than I $\mathrm{mm}$ in thickness. The interior of the dishes presents a surface area of 54 square centimeters.

Another dish has been designed which is hemispherical in shape with the exception of a flattened portion of $3 \mathrm{~cm}$ in diameter on its bottom. Its dimensions are $9.5 \mathrm{~cm}$ in diameter across the top and $4.5 \mathrm{~cm}$ deep. The hemispherical shape has the advantage of affording the greatest capacity for the least wall area. As, of course, the weight of the dish lies in its walls, the smaller the area of the walls the less will be the weight. The hemispherical dish therefore gives the greatest capacity for the least weight.

The first experiments were made to determine the most satisfactory method for drying, attention being paid to speed as well as to accuracy. A method frequently used in drying the Classen dish with deposited metal is to wash with absolute alcohol and to burn off the excess. Applying the severest test first, this method of drying was used; the dishes were washed with distilled water, followed by absolute alcohol and the excess of alcohol was burnt off. After cooling in a desiccator the dishes were weighed.

Some of the results showed only a slight loss in weight while others showed a loss of almost $5 \mathrm{mg}$, due, no doubt, 
as was found, to the loss in paraffine through being dissolved by the alcohol.

The method was then adopted of drying, after washing with distilled water, by holding the dishes by means of crucible tongs directly over the smokeless flame of a Bunsen burner, moving them around during the drying so that the heating would be uniform; they were then cooled in a desiccator. This extremely crude method was justified by its rapidity and gave surprisingly satisfactory results, as is shown by Table I.

\section{TABLE I}

\begin{tabular}{c|c|c|c} 
Number & Weight before & Weight after & Loss \\
\hline I & 37.2660 & 37.2660 & None \\
2 & 37.2660 & 37.2658 & 0.0002 \\
3 & 37.2658 & 37.2652 & 0.0006 \\
4 & 33.1400 & 33.1400 & None \\
5 & 33.1400 & 33.1400 & $*$ \\
6 & 33.1400 & 33.1400 & $*$
\end{tabular}

A number of determinations of efficiency were then made by electrolyzing an acidified solution of copper sulphate contained in a graphite dish in series with a copper coulometer filled with a portion of the same solution. Copper anodes were employed in both cells and a copper cathode in the coulometer. A current of O.I-O.I6 amperes was passed and the electrolysis was continued for about one hour in each run. Some of the results obtained are given in Table II.

TABLE II

\begin{tabular}{c|ccc} 
Number & $\begin{array}{c}\text { Cathode gain in } \\
\text { coulometer }\end{array}$ & $\begin{array}{c}\text { Cathore gain in } \\
\text { graphite dish }\end{array}$ & Difference \\
\hline 1 & 0.1140 & 0.1128 & 0.0012 \\
2 & 0.1582 & 0.1570 & 0.0012 \\
3 & 0.1784 & 0.1770 & 0.0014 \\
4 & 0.1748 & 0.1728 & 0.0020 \\
5 & 0.2008 & 0.2004 & 0.0004 \\
6 & 0.1924 & 0.1920 & 0.0004 \\
7 & 0.1924 & 0.1924 & 0.0000 \\
8 & 0.1772 & 0.1758 & 0.0012 \\
9 & 0.1818 & 0.1802 & 0.0016 \\
10 & 0.2126 & 0.2122 & 0.0004
\end{tabular}


An occasional result failed signally to agree with the theoretical value. As a check on the method, a Classen dish, containing a similar solution and copper anode was also placed in the circuit; this cell was electrolyzed in series with the other two. The platinum dish was dried with alcohol, the excess of which was burnt off, and was cooled in a desiccator. The results gotten with the Classen dish are shown in Table III.

TABLE III

\begin{tabular}{c|cc|c}
\hline Number & $\begin{array}{c}\text { Cathode gain in } \\
\text { coulometer }\end{array}$ & $\begin{array}{c}\text { Cathode gain in } \\
\text { Classendish }\end{array}$ & Difference \\
\hline I & 0.1140 & 0.1142 & 0.0002 \\
2 & 0.1084 & 0.1040 & 0.0044 \\
3 & 0.1748 & 0.1768 & 0.0020 \\
4 & 0.2008 & 0.2030 & 0.0022 \\
5 & 0.1924 & 0.1919 & 0.0005 \\
6 & 0.1772 & 0.1753 & 0.0019 \\
7 & 0.1818 & 0.1807 & 0.0011
\end{tabular}

These results, as a whole, are hardly as good as those obtained with the graphite dish and, like those, an occasional one was bad. The errors observed in the case of the Classen dish are doubtless due to hurried and perhaps careless manipulation. It may be noticed, moreover, that in the case of the graphite dish the results are mostly low, while in that of the platinum dish they are sometimes high and sometimes low.

In Table IV the results obtained with the Classen dish are tabulated against those obtained with the graphite dish. The results obtained for the graphite dish as set forth in this table are for the most part likewise lower than those obtained with the platinum dish. It appears that there is perhaps some loss in the weight of the dish itself in drying which causes the low results. Therefore, the method of drying directly over the flame will be abandoned and instead the dishes will be washed thoroughly with distilled water and then dried rapidly in a stream of warm, dry air. 
TABLE IV

\begin{tabular}{c|c|c|c}
\hline Number & $\begin{array}{c}\text { Cathode gain in } \\
\text { platinum dish }\end{array}$ & $\begin{array}{c}\text { Cathode gain in } \\
\text { graphite dish }\end{array}$ & Difference \\
\hline I & 0.1142 & 0.1128 & 0.0014 \\
2 & 0.1040 & 0.1038 & 0.0002 \\
3 & 0.1650 & 0.1642 & 0.0008 \\
4 & 0.1768 & 0.1728 & 0.0040 \\
5 & 0.2030 & 0.2031 & 0.0001 \\
6 & 0.1919 & 0.1920 & 0.0001 \\
7 & 0.1997 & 0.1974 & 0.0023 \\
8 & 0.1753 & 0.1758 & 0.0005 \\
9 & 0.1807 & 0.1802 & 0.0005 \\
IO & 0.2125 & 0.2122 & 0.0003
\end{tabular}

The dishes are now being tested in actual analyses. Known volumes of a standard copper solution are being analyzed in them with rotating anode and at high current density. This will be considered the crucial test and not until the results of this test are known can the dish be unqualifiedly recommended. This paper is therefore merely a preliminary note. No further tests are needed, however, we feel, to show that it may be substituted profitably in numerous electrochemical operations where the Classen dish has been employed heretofore, or would have been had it been a less expensive article.

These dishes have been suggested for use in other operations not connected with electrolysis, such as digestions in hydrofluoric acid at high temperatures. In such cases the beaker shape would probably be advantageous.

A rotating graphite anode has been designed to accompany the dishes. This is constructed after the general plan of the platinum anode described by Miss Langness. ${ }^{1}$ It is dish-shaped and contains radial slits in its walls and a circular opening in its bottom. It is hoped that by means of it the interesting results obtained with the anode referred to above may be duplicated.

Work is now being carried on, as intimated above, to

${ }^{1}$ Jour. Am. Chem. Soc., 29, 459 (1907). 
show the limits of the graphite cathode dish. ${ }^{1}$ A further report therefore will be made. The efficiency of the dish in numerous electrochemical separations and operations will also be determined. It is also our intention to investigate the rotating dish anode referred to above and a smaller, discshaped, rotating anode with a graphite stem.

\section{Summary}

(I) Simple, graphite electrodes are described which are designed for use in the place of platinum as insoluble electrodes in electrochemical experiments.

(2) An account is given of preliminary experiments with a graphite dish intended to supplant in some forms of electrochemical analysis the Classen platinum dish.

(3) The dish is recommended for electrochemical separations and for analyses where great accuracy is not required. Confidence is had that, after it has been further developed, it will be able to take the place entirely of the platinum cathode dish.

ITeslegan Lniversity,

April I, I909.

1 Manufactured and on sale by Eimer \& Amend, New York. 